\title{
Original
}

\section{Cáncer de pene. Nuestra experiencia en 15 años}

\author{
Carlos Di Capua Sacoto, Sátur Lujan Marco, Gonzalo Morales Solchaga, Alberto Budía Alba, \\ José L. Pontones Moreno, Juan F. Jiménez Cruz
}

Servicio de Urología. Hospital Universitario La Fe. Valencia, España

\begin{abstract}
Resumen
Objetivos: Revisión y análisis de los factores de riesgo, diagnóstico, tratamiento y evolución del cáncer de pene.

Material y Métodos: Realizamos un estudio descriptivo retrospectivo desde 1992 hasta el 2007, en el que se incluyeron 47 pacientes con diagnóstico de cáncer de pene tratados en nuestro servicio.

Se analiza factores de riesgo, sintomas, diagnóstico, tratamiento, y evolución de los pacientes. El estadiaje clínico y anatomopatológico se hizo acorde a la clasificación TNM, teniendo en cuenta el examen físico, pruebas de diagnóstico por imagen y hallazgos de la pieza quirúrgica. El tiempo medio de seguimiento es de 50 meses (rango 12 a 120 meses).

Para el análisis estadístico univariante se utilizo la prueba de Chi-cuadrado, y para el análisis de la supervivencia el método de Kaplan Meir. Resultados: La edad media de los pacientes fue de 60 años (28-91 años), siendo el 95\% de ellos mayores de 50.

Presentaron adenopatías al diagnóstico 16 pacientes (34\%) siendo las más frecuente (62\%) las adenopatías unilaterales inguinales. Ningún caso presentó metástasis a distancia.

De los 27 casos (57\%) a los que se les realizó calcio en sangre al momento del diagnostico, encontramos hipercalcemia en 8 (30\%). El tratamiento fue quirúrgico en la mayoría (95\%), siendo la técnica más empleada la penectomía parcial en 25 (53\%). En 4 sujetos se decidió la radioterapia como tratamiento inicial. A 14 se les realizó una linfaadenectomía, siendo la técnica más frecuente la inguinal bilateral profunda (8 pacientes). Respecto al estadio TNM, la mayoría de pacientes presentaron un estadio clínico localizado: T1NOMO 23 \%, T2NOMO 27\%. El diagnóstico anatomopatológico en el 100\% de los casos fue carcinoma epidermoide (80\% fue bien diferenciado)

En la última revisión 31 casos (76\%) estaban libres de enfermedad. Hubo 11 (24\%) casos que presentaron recidiva y fueron tratados: 3 con radioterapia, 7 cirugía de rescate y 1 de ellos con quimioterapia.

Los factores pronósticos adversos más importantes son la presencia de adenopatías, el estadio clínico al diagnóstico y el grado de diferenciación celular $(\mathrm{p}=0,001)$

Once pacientes fallecieron a lo largo del seguimiento, 9 de los cuales por progresión de la enfermedad.

Conclusiones: El carcinoma de pene sigue siendo una enfermedad maligna poco frecuente en nuestro medio. Los factores pronósticos adversos más importantes son la presencia de adenopatías, el estadio clínico al diagnóstico y el grado de diferenciación celular. El tratamiento más eficaz del cáncer de pene es quirúrgico, reservando la quimioterapia y radioterapia para adyuvancia o terapia de rescate. La hipercalcemia es un hallazgo frecuente cuando los pacientes presentan adenopatías.
\end{abstract}

Palabras clave: Cáncer. Pene. Supervivencia.

\section{Cancer of the penis. Our experience in 15 years}

\section{Abstract}

Objective: To review and analysis the diagnosis, treatment, evolution, and risk factors of the penis cancer.

Material and Methods: We carried out a retrospective study between 1992 and 2007 . We included 47 patients with penis cancer diagnose of treated in our service. We analyzed risk factors, symptoms, diagnosis and treatment. The staging was done according to the TNM classification, taking into account the physical exam, radiology and the surgical findings.

The average time of follow-up was 50 months (range 12 to 120 months).

For univariate statistical analysis the Chi-square test was used, and for the survival the method of Kaplan Meir.

Results: Mean age was 60 years (28-91 years), being 95\% older than 50 .

At the diagnosis 16 patients (34\%) had lymphatic nodes, being the most frequent location was unilateral inguinal nodes (62\%).

Of the 27 cases (57\%) who underwent blood calcium check at the time of diagnosis, we found hypercalcaemia in 8 patients (30\%).

The surgical treatment was the gold standard treatment. The technique more frequently employed was the partial penectomy 25 (53\%). 4 patients received radiotherapy as inicial treatment. Lymphadenectomy was performed in 14 patients, mostly bilateral inguinal lymphadenectomy (8 patients).

The pathology finding at $100 \%$ of the cases was squamous cell carcinoma ( $80 \%$ well differentiated). Regarding to the TNM, most of the patients had a clinical stage located: T1NOMO 23\%, T2NOMO 27\%.

The most significant adverse prognostic factors were the presence of lymph nodes, clinical stage at the diagnosis and the degree of cell differentiation $(\mathrm{p}=0001)$.

At the last review $36(76 \%)$ cases were free of disease. There were $11(24 \%)$ cases that presented recurrence and were treated: 3 patients with radiotherapy, surgery was used in 7 patients and 1 patient with chemotherapy. Eleven patients died during follor up, 9 of thes from disease progression.

Conclusions: The carcinoma of the penis remains a rare malignancy in our centre. Hypercalcemia is a common finding when patients have lymph nodes. The most significant adverse prognostic factors were the presence of lymph nodes, clinical stage at the diagnosis and the degree of cell differentiation. The most effective treatment for cancer of the penis is surgery reserving chemotherapy and radiation therapy for the recurrence.

Keywords: Cancer. Penis. Surveillance. 
$\mathrm{E}$ 1 carcinoma de pene es una enfermedad maligna poco frecuente en países desarrollados, 0,10,7 por 100.000 habitantes. En España, su tasa de prevalencia se sitúa en el $0,7 \%$ de los tumores malignos del varón ${ }^{1-3}$. Sin embargo, en países subdesarrollados la incidencia se incrementa considerablemente hasta alcanzar el 10-20\% de los tumores malignos en el varón ${ }^{3,4}$.

La fimosis y los procesos irritativos crónicos relacionados con una higiene deficiente se asocian al desarrollo del cáncer de pene, mientras que, según algunos autores la circuncisión neonatal proporciona protección contra este tumor ${ }^{1,2,4}$. Actualmente se evidencia una relación directa entre la infección por papilomavirus de los serotipos 16 y 18 con el carcinoma epidermoide de pene siendo ésta particularmente estrecha en casos de carcinoma in situ. La infección por virus papiloma humano (HPV) y la exposición a productos del tabaco se asocia a desarrollo de cáncer de pene ${ }^{5}$. El serotipo 16 HPV es el tipo detectado con mayor frecuencia en carcinoma primario $^{6,7}$. Además se sabe que la infección por HPV se correlaciona directamente con el número de parejas sexuales, lo que también se ha relacionado con el cáncer de pene ${ }^{8}$.

Con respecto a la circuncisión como prevención en el cáncer de pene, es un aspecto controvertido, ya que no existen datos suficientes para recomendarla de forma sistemática ${ }^{9}$.

Los tumores de pene se presentan, en nuestro medio, más frecuentemente entre la quinta y sexta década de vida, con un pico en la octava ${ }^{5}$ La localización puede ser en todo el pene sin embargo, lo más frecuente, por orden de frecuencia suele ser glande, prepucio, surco balanoprepucial, y cuerpo de pene $e^{10,11}$. En la mayoría de ocasiones las lesiones se suelen limitar al pene.

El cáncer de pene se asocia a hipercalcemia. La hipercalcemia se relaciona con la extensión de la enfermedad, por lo general con la presencia de adenopatías. Se sabe que el tumor y las metástasis, tanto en ganglios como en otros órganos producen hormona paratiroidea (PTH) que activa la reabsorción ósea mediante un aumento de la actividad osteoclástica en el hueso ${ }^{12,13}$.

Actualmente el TNM da importancia al compromiso ganglionar como factor pronóstico de supervivencia, ya que la supervivencia de pacientes con adenopatías es menor que en pacientes sin adenopatias y cuando son unilaterales mayor que si son bilaterales. La presencia y el número de adenopatías en la región inguinal son los factores pronósticos de supervivencia más importantes ${ }^{5,14}$.

El tratamiento de elección en el cáncer de pene es el quirúrgico, siendo especialmente efectivo en los tumores localizados, con tasas de recurrencia local del $8 \%{ }^{11,15}$. En los tumores de $4 \mathrm{~cm}$ o mayores o que invaden los cuerpos cavernosos o uretra, la penectomía parcial o total es el tratamiento de elección. Los enfermos con tumor peneano primario localizado, $\mathrm{y}$ con buena diferenciación celular tienen bajo riesgo de metástasis. Estos pacientes se pueden tratar con procedimientos conservadores del pene o glande ${ }^{16}$.

\section{MATERIAL Y MÉTODOS}

Hemos realizado un estudio descriptivo retrospectivo desde 1992 hasta el 2007 donde se incluyen 47 pacientes con diagnóstico de cáncer de pene tratados en nuestro servicio. El tiempo medio de seguimiento fue de 50 meses, con un rango de 12 a 120 meses.

Los datos se recogieron de la historia clínica. Se analizaron factores de riesgo, síntomas, procedimientos diagnósticos, tratamiento, y evolución.

Se realizó a todos nuestros pacientes examen físico, radiografía de tórax, tomografía computarizada abdomino-pélvica (TC) y en algunos casos calcio en sangre.

El estadiaje se hizo acorde a la clasificación TNM, teniendo en cuenta el examen físico, pruebas de diagnóstico por imagen y hallazgos de la pieza quirúrgica.

\section{RESULTADOS}

La edad media en el momento del diagnóstico fue de 60 años (28-91 años). 45 pacientes (95\%) eran mayores de 50 años.

En nuestra serie 20 pacientes (42\%) eran fumadores y 27 no fumadores (58\%).

El 95\% (45 pacientes) no estaban circuncidados y solo 2 (5\%) fueron circuncidados en la edad adulta. Presentaban algún grado de fimosis 21 pacientes (46\%) y sólo tenían como antecedentes enfermedades de transmisión sexual. Dentro de los pacientes no circuncidados (45), 3 pacientes $(6,66 \%)$ presentaban una balanitis xerotica. La misma que en el momento de la cirugía fue solucionada.

La forma de presentación más frecuente fue una lesión exofítica o verrucosa en 31 pacientes (66\%), una lesión ulcerada en 11 pacientes $(23,4 \%)$ y en forma de nódulo o masa en 5 (10,6\%) (Tabla 1). 
Tabla 1. Datos descriptivos de la serie

\begin{tabular}{|c|c|}
\hline Edad (media) & 66 años (28-91) \\
\hline \multicolumn{2}{|l|}{ Fumadores } \\
\hline Sí & $20(42,6 \%)$ \\
\hline No & $27(57,4 \%)$ \\
\hline \multicolumn{2}{|l|}{ Fimosis } \\
\hline Sí & $25(53,2 \%)$ \\
\hline No & $22(46,8 \%)$ \\
\hline \multicolumn{2}{|l|}{ Localización } \\
\hline Glande & $19(40.4 \%)$ \\
\hline Prepucio & $16(34 \%)$ \\
\hline Surco balanoprepucial & $8(17 \%)$ \\
\hline Masa genital & $3(6,4 \%)$ \\
\hline Cuerpo de pene & $1(2,1 \%)$ \\
\hline \multicolumn{2}{|l|}{ Forma de Presentación } \\
\hline Exofítica o verrucosa & $31(66 \%)$ \\
\hline Ulcerativa & $11(23,4 \%)$ \\
\hline Bulto & $5(10,6 \%)$ \\
\hline \multicolumn{2}{|l|}{ Adenopatías } \\
\hline Sí & $16(34 \%)$ \\
\hline No & $31(66 \%)$ \\
\hline Calcio mg/dl (media) & $10,1(8,7-15,20)$ \\
\hline \multicolumn{2}{|l|}{ Estadio Clínico (TNM) } \\
\hline Estadio I & $11(23 \%)$ \\
\hline Estadio II & $14(29 \%)$ \\
\hline Estadio III & $18(38 \%)$ \\
\hline Estadio IV & $4(8,5 \%)$ \\
\hline \multicolumn{2}{|l|}{ Grado Histológico } \\
\hline Bien diferenciado & $36(76 \%)$ \\
\hline Moderadamente diferenciado & $6(12,8 \%)$ \\
\hline Mal diferenciado & $3(6,4 \%)$ \\
\hline Indiferenciado & $2(4,3 \%)$ \\
\hline \multicolumn{2}{|l|}{ Tratamiento inicial } \\
\hline Circuncisión & $10(21 \%)$ \\
\hline Penectomia Parcial & $25(53 \%)$ \\
\hline Penectomia Total & $8(17 \%)$ \\
\hline Radioterapia & $4(8,5 \%)$ \\
\hline
\end{tabular}

La localización más frecuente fue en glande 19 pacientes $(40,4)$, seguida de prepucio 16 pacientes (34\%), surco balanoprepucial en 8 (17\%), masa que engloba área genital en $3(6,4 \%)$ y cuerpo de pene en 1 paciente $(2,1 \%)$.

Presentaron adenopatías al diagnóstico 16 pacientes (34\%) de las cuales 10 (64\%) tenían adenopatías palpables a nivel inguinal y en 6 (36\%) se observaron las mismas al realizar la TC. Nueve pacientes (62\%) presentaban adenopatías inguinales unilaterales, 4 bilaterales (24\%) y 2 obturatrices (12\% de los casos). Ningún caso presentó metástasis a distancia en el momento del diagnóstico.
De los 27 sujetos (57\%) a los que se les realizó calcio en sangre al momento del diagnóstico, los niveles eran normales en 19 pacientes (70\%) y presentaban hipercalcemia en 8 (30\%); de estos ocho pacientes, 6 presentaban adenopatías mientras que en 2 no se hallaron. De los 19 pacientes con normocalcemia ninguno tenían adenopatías.

El tratamiento en la mayoría de los casos (90\%) fue quirúrgico. Las técnicas empleadas fueron circuncisión (tumores prepuciales) en 10 sujetos (21\%), penectomía parcial en 25 (53\%), y penectomía total en 8 (17\%). En 4 sujetos se decidió la radioterapia como tratamiento inicial.

De los 16 pacientes con adenopatías al diagnostico a 14 se les realizó linfadenectomía. A 8 linfadenectomía inguinal bilateral profunda, a 2 inguinal unilateral profunda y 4 inguinal unilateral superficial.

El diagnóstico anatomopatológico en el $98 \%$ de los casos fue carcinoma epidermoide. En un $76.6 \%$ el tumor fue bien diferenciado, en $12,8 \%$ moderadamente diferenciado y en un 7,3\% indiferenciado. En las muestras se evidenciaron lesiones premalignas, en $7 \%$ de ellas se observo liquen escleroso y en $5 \%$ se identifico liquen atrófico. Un paciente $(2 \%)$ presentó una variedad de tumor verrucoso de BuscheLowestein.

Cuando se agrupo el TNM por estadios encontramos: estadio I en 11 pacientes $(23,4 \%)$, estadio II en 14 (29,8\%), estadio en III 18 (38,3\%) y estadio IV en 4 pacientes $(8,5 \%)$. Se consideró estadio localizado a los estadios I y II, mientras no localizados los estadios III y IV.

En la última revisión 36 (76\%) pacientes estaban libres de enfermedad y 11 (24\%) habían presentado recidiva. De los pacientes que recidivaron, 3 pacientes recibieron radioterapia como tratamiento inicial del tumor, a 2 se les realizó una circuncisión, en 5 se efectuó una penectomía parcial y en 1 una penectomía radical. Con respecto a la localización de la recidiva, en 6 de ellos fue a nivel de pene y 5 pacientes presentaron adenopatías inguinales palpables. Once de estos fallecieron durante su seguimiento, nueve de los cuales por progresión de la enfermedad (Tabla 2).

Se realizo un análisis univariante mediante la prueba de chi cuadrado donde se observo que aquelos pacientes que presentaban un estadío mas avanzado y adenopatías al diagnóstico tenían menor supervivencia que los estadios localizados, siendo esta 
Tabla 2. Supervivencia de los pacientes

\begin{tabular}{lc}
\hline Estado & $\mathbf{N}^{\mathbf{0}}$ (\%) \\
\hline Vivos & $36(76)$ \\
Recidivas & $16(34)$ \\
Muertos & $11(23)$ \\
Muertos cáncer específica & $9(19)$ \\
\hline
\end{tabular}

diferencia estadísticamente significativa ( $\mathrm{p}=0,0001)$; además, aquellos pacientes que presentaban un grado histológico mas indiferenciado tenían peor supervivencia ( $\mathrm{p}=0,0001)$. Con respecto al calcio, en pacientes que presentaban valores más elevados de calcio tenian una tendencia a una menor supervivencia, aunque estas diferencias solo se aproximaban a la significación estadística ( $\mathrm{p}=0,070)$ (Tabla 3). La hipercalcemia se correlaciono con el hallazgo al momento del diagnóstico de adenopatías palpables, si bien esta correlación no era estadísticamente significativa. El ser fumador no se implicó con una menor supervivencia, tampoco se asocio a un estadio más avanzado, ni con un grado histológico más desfavorable.

Tabla 3. Resultados del análisis univariante

\begin{tabular}{lc}
\hline Variable & Valor de p \\
\hline Fumador & 0,439 \\
Grado Histológico & 0,001 \\
TNM (clasificado según estadio) & 0,001 \\
Adenopatías & 0,001 \\
Calcio & 0,070 \\
\hline
\end{tabular}

Las recidivas aparecieron con mayor frecuencia en pacientes que recibieron como tratamiento inicial la radioterapia $(60 \%)$.

Se analizó la supervivencia de los pacientes mediante el método de Kaplan Meir, y en nuestra serie observamos que un $65 \%$ de los pacientes sobrevive a los 5 años. La media de supervivencia actuarial en nuestra serie fue de 67 meses (rango 53 a 97 meses) (Fig. 1).

\section{DISCUSIÓN}

El cáncer de pene en España y en nuestro centro es poco frecuente. En nuestra serie la edad media en el momento del diagnóstico fue de 60 años (28 y 91 años); 45 pacientes (95\%) eran mayores de 50 años, estos datos son similares a los aportados por

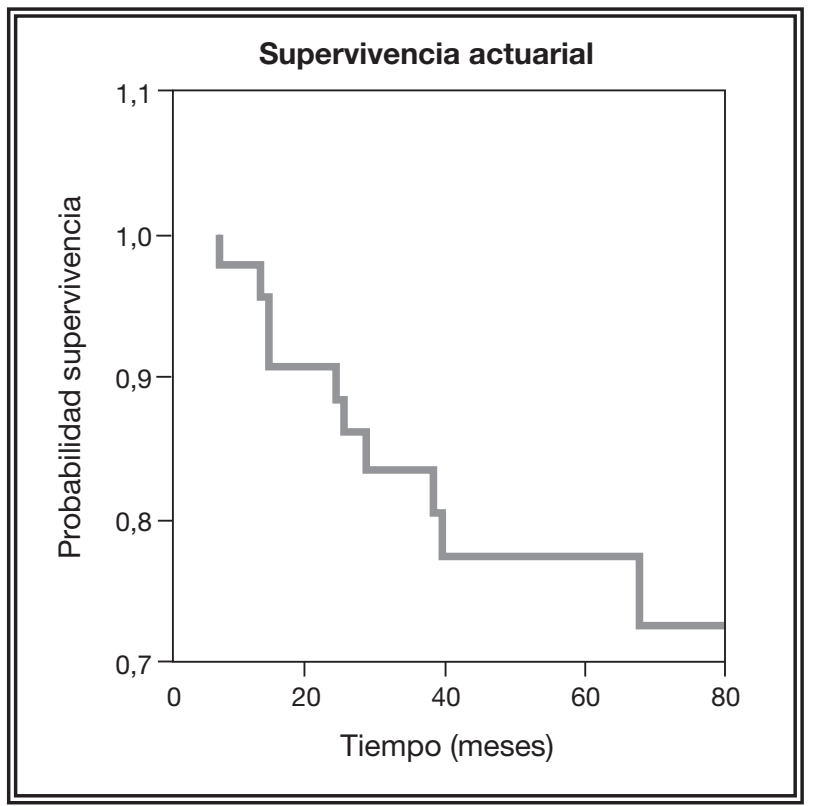

FIGURA 1. Curva de supervivencia mediante el método de Kaplan Meir.

otros trabajos tanto en nuestro medio como en Europa, donde se observa que el cáncer de pene aumenta en prevalencia e incidencia a medida que aumenta la edad ${ }^{1,2,4,17}$.

La localización más frecuente de las lesiones es a nivel de glande (40.4\%) y prepucio (34\%), la forma de presentación más frecuente es una lesión exofítica o verrucosa (66\%) siendo poco frecuente las lesiones ulcerativas $(23 \%)$. En una serie de pacientes diagnosticados de cáncer de pene en España de Soto $\mathrm{M}$ et $\mathrm{al}^{4}$. Se recoge que la localización más frecuente de la lesión inicial fue el glande (50\%) seguida del prepucio $(22,2 \%)$, surco balanoprepucial $(16,7 \%)^{4}$.

Y su forma de presentación más habitual era: lesión vegetante en el pene (50\% de los casos), fimosis muy cerradas con supuración, prepucial $(38,8 \%)$ y masa inguinal $(11,2 \%)^{4}$.

En el momento del diagnóstico 16 pacientes (34\%) presentaron adenopatías palpables o que se diagnosticaron mediante alguna técnica e imagen. Estos datos son similares a otras series clásicas y recientes del cáncer de pene ${ }^{17,18}$.

De nuestros pacientes un $42,6 \%$ eran fumadores. Ninguno tenía antecedentes de circuncisión durante la edad pediátrica. Un total de 21 (46\%) pacientes presentaba algún grado de fimosis. Todos estos datos sugieren que en nuestra población sigue siendo poco frecuente la circuncisión durante la 
edad pediátrica y que los principales factores de riesgo como el tabaco y la fimosis son frecuentes en los pacientes diagnosticados de cáncer de pene ${ }^{1,4,14}$.

En relación con el calcio en sangre y las adenopatías encontramos que en un 30\% (8) de los pacientes existe hipercalcemia y de éstos 6 (75\%) presentaban adenopatías; nuestros datos son similares a los de otros trabajos, donde se ha observado la capacidad que tiene el cáncer de pene para producir PTH y en consecuencia aumenta la actividad oasteoclástica ósea incrementando los niveles de calcio en sangre ${ }^{12,13,19}$. Si bien en nuestro protocolo no se contemplaba solicitar calcio en sangre en el momento del diagnóstico, dado los hallazgos de este estudio, donde es frecuente que los pacientes con adenopatías presenten hipercalcemia, y los datos de la literatura, pensamos que es necesario solicitarlo a todos los pacientes en el momento del diagnóstico.

El tratamiento en la mayoría de los casos (90\%) fue quirúrgico. Las técnicas empleadas fueron circuncisión en 10 pacientes (21\%), penectomía parcial en 25 (53\%), y penectomía total en 8 (17\%). En 4 pacientes se decidió la radioterapia como tratamiento inicial. En nuestro servicio no hemos realizado técnicas mínimamente invasivas como la cirugía de Mohs o fotocoagulación con láser, aunque son técnicas descritas para el tratamiento del cáncer de pene localizado ${ }^{20-23}$.

Existen diversos trabajos publicados por Cubillas et al. ${ }^{24-26}$ donde describe 5 patrones de crecimiento del cáncer de pene: extensivo superficial, crecimiento vertical, verruciforme, múlticentrico y mixto. Todos estos crecimiento al ser analizados demostraron que existía una correlación con la supervivencia. Siendo los de crecimiento vertical y múlticentricos los de peor pronóstico.

Con respecto a la linfadenectomía es un aspecto que continua siendo controvertido, sobre todo en cuanto al momento en el que realizarla, si debe ser linfadenectomía unilateral o bilateral y si profunda o superficial. En nuestra serie hemos realizado a 14 de los 16 pacientes que presentaron adenopatías una linfadenectomía. A 2 pacientes no se les realizo linfadenectomía por la edad (82 y 83 años respectivamente). A 8 pacientes se les practicó lifadenectomía inguinal bilateral profunda, a 2 inguinal unilateral profunda y a 4 inguinal unilateral superficial. La complicación más frecuente que apareció después de la linfadenectomía fue el linfedema, en 6 pacientes (42\%). Además más habitual cuanto ma- yor era la extensión de la linfadenectomía siendo más intensa en los que se les realizo una linfadenectomía profunda. Actualmente existen datos que avalan la linfadenectomía profunda en los pacientes con una adenopatía inguinal palpable en el momento del diagnóstico ${ }^{27-29}$. Aunque se han ensayado técnicas para el diagnóstico de las adenopatías como el estudio del ganglio centinela o la PAAF de ganglio aun no existen datos suficientes para recomendar el uso rutinario de ninguna de estas técnicas ${ }^{29,30}$.

El tratamiento mediante penectomía parcial o total suele ser efectiva con pocas recidivas a nivel local. Sin embargo en el momento de la cirugía pueden existir micrometástasis a nivel ganglionar que posteriormente influirán en la aparición de las recidivas loco-regionales y a distancia.

El aspecto más controvertido en cuanto al tratamiento de las adenopatías continua siendo cuando tratarlas. ¿Se debe realizar una cirugía inmediata o una cirugía diferida?

La morbilidad que produce la linfadenectomía es el principal argumento en contra de su práctica sistemática. Sin embargo seis series revelan una mayor supervivencia en pacientes tratados de forma temprana frente a los tratados de forma diferida. Por ejemplo, Lont et al. ${ }^{24}$ informan que la linfadenectomía adyuvante inmediata permitió una supervivencia libre de la enfermedad a 5 años en 6 de 8 pacientes con ganglios positivos $(75 \%)$, en comparación con 1 de 12 (8\%) en los tratados con linfadenectomía diferida. Kroon et al. ${ }^{33}$ compararon la supervivencia de 20 pacientes con ganglios positivos detectados según la técnica de ganglio centinela con la de 20 pacientes con disección ganglionar diferida. La supervivencia a 3 años en los pacientes detectados durante el seguimiento exhaustivo fue de solo el $35 \%$ en comparación con el 84\% del grupo de ganglio centinela $(\mathrm{p}=0,001)^{5,30} \mathrm{Y}$ cinco de las seis series muestran que la disección diferida rara vez evita que el paciente presente una recurrencia.

También encontramos, en el estudio de Johnson y $\mathrm{Lo}^{34}$ que en 101 linfadenectomías tienen $50 \%$ de linfedema y 0 muertes. En una serie más actual, BevanThomas et al. ${ }^{30}$ en 106 disecciones observaron un 23 $\%$ de linfedemas y un 1,8\% de muertes secundarias a la linfadenectomía ${ }^{5,27,31}$. Entre estas dos series existe diferencias estadísticamente significativas $(p=0,001)$ en cuanto a la aparición de linfedema ${ }^{5}$. En la nuestra de 14 linfadenectomías, 6 (42\%) presentaron linfedema, estos datos son similares a las series publicadas. 
En nuestra serie se observó que aquellos pacientes que presentaban un estadio más avanzado y adenopatías al diagnóstico tenían peor supervivencia que estadios localizados, siendo la diferencia estadísticamente significativa $(\mathrm{p}=0,0001)$; además, aquellos pacientes que presentaban un tumor más indiferenciado tenían peor supervivencia $(p=0,0001)$. Estos datos son similares a los encontrados por otros grupos de trabajo ${ }^{1,5,16,17,24}$.

\section{CONCLUSIONES}

El carcinoma de pene sigue siendo una enfermedad maligna poco frecuente en nuestro medio. La hipercalcemia es un hallazgo frecuente en nuestra serie cuando los pacientes presentan adenopatías.

Los factores pronósticos adversos más importantes son la presencia de adenopatías, el estadio clínico al diagnóstico y el grado de diferenciación celular. El tratamiento más eficaz del cáncer de pene es quirúrgico, reservando la quimioterapia y radioterapia para adyuvancia o terapia de rescate

\section{REFERENCIAS}

1. Solsona E. Cáncer de pene. Actas Urol Esp. 2002;26(8):525-531.

2. Rippentrop JM, Joslyn SA, Konety BR. Squamous cell carcinoma of the penis: evaluation of data from the surveillance, epidemiology, and end results program. Cancer. 2004;101(6):1357-1363.

3. Peyrí Rey E. Prevención del cáncer de pene. Actas Urol Esp. 2008;32(4):470471 .

4. Soto Delgado M, Arredondo Martínez F, Pedrero Márquez G, Basquero González B, Zurera Cosano A, Linares Armada R. Cáncer de pene. Una revisión de 18 casos. Actas Urol Esp. 2003;27(10):797-802.

5. Donald F. Lynch and Curtis A. Pettaway. Tumors of the penis. Cambell of Urology. Walsh P, Retik A, Vaughan E, Wein A, Kavossi L, Novick A, Partin A. and Peters C. 8 edición, 2004;3225-3265.

6. Wiener JS, Walther PJ. The association of oncogenic human papillo-maviruses with urologic malignancy. Surg Oncol Clin North Am. 1995;4(2):257-276.

7. Boshart M, zur Hausen H. Human papillomaviruses (HPV) in BuschkeLöwenstein tumors: Physical state of the DNA and identification of a tandem duplication in the noncoding region of a human papillomavirus 6 subtype. J Virol. 1986;58(3):963-966.

8. Castellsagué X, Ghaffari A, Daniel RW, Bosch FX, Muñoz N, Shah KV. Prevalence of penile human papillomavirus DNA in husbands of women with and without cervical neoplasia: a study in Spain and Colombia. J Infect Dis. 1997; 176(2):353-361

9. Shapiro E. American Academy of Pediatrics policy statements on circumcision and urinary tract infection. Rev Urol. 1999;1(3):154-156.

10. Cruz Guerra NA, Allona Almagro A, Clemente Ramos L, Linares Quevedo A, Briones Mardones G, Escudero Barrilero A. Linfadenectomía en el carcinoma escamoso de pene: revisión de nuestra serie. Actas Esp Urol. 2000;24(9):709-714

11. Solsona E, Algaba F, Horenblas S, Pizzocaro G, Windahl T; European Association of Urology. EAU guidelines on penile cancer. Eur Urol 2004;46(1):1-8.

12. Sklaroff RB, Yagoda A. Penile cancer: Natural history and therapy. In Spiers ASD, ed: Chemotherapy and Urological Malignancy. New York, SpringerVerlag, 1982:98-105.

13. Malakoff AF, Schmidt JD. Metastatic carcinoma of penis complicated by hypercalcemia. Urology. 1975;5(4):510-513.

14. Rubio-Briones J, Villavicencio H, Regalado R, Chéchile G, Algaba F, Monreal $\mathrm{F}$, et al. Carcinoma escamoso de pene; protocolo de tratamiento según nuestra experiencia en 14 años. Arch Esp Urol. 1997;50(5):473-480.
15. Leijte JA Kirrander P, Antonini N, Windahl T, Horenblas S. Recurrence patterns of squamous cell carcinoma of the penis: recommendations for followup based on a two-centre analysis of 700 patients. Eur Urol. 2008;54(1) 161-169.

16. Solsona E, Iborra I, Rubio J, Casanova JL, Ricós JV, Calabuig C. Prospective validation of the association of local tumor stage and grade as a predictive factor for occult lymph node micrometastasis in patients with penile carcinoma and clinically negative inguinal lymph nodes. J Urol. 2001;165(5): 1506-1509.

17. Ornellas AA, Seixas AL, Marota A, Wisnescky A, Campos F, de Moraes JR. Surgical treatment of invasive squamous cell carcinoma of the penis: Retrospective analysis of 350 cases. J Urol. 1994;151(5): 1244-1249.

18. Puras-Baez A, Rivera-Herrera J, Miranda G, et al: Role of superficial inguinal lymphadenectomy in carcinoma of the penis. J Urol. 1995;153:246.

19. Anderson EE, Glenn JF. Penile malignancy and hypercalcemia. JAMA. 1965; 192:328-329.

20. Bleeker MC, Heideman DA, Snijders PJ, Horenblas S, Dillner J, Meijer CJ. Penile cancer: epidemiology, pathogenesis and prevention. World J Urol. 2008;8.

21. Cuevas J, de Eusebio E, Diez E, Castiñeira I. Mohs micrographic surgery: aplication of this technique to penile neoplasms. Actas Urol Esp. 2007 31(9):1076-1081.

22. Meijer RP, Boon TA, van Venrooij GE, Wijburg CJ. Long-term follow-up after laser therapy for penile carcinoma. Urology. 2007;69(4):759-762.

23. Mohs FE, Snow SN, Larson PO. Mohs micrographic surgery for penile tumors. Urol Clin North Am. 1992;19(2):291-304.

24. Velazquez EF, Ayala G, Liu H, Chaux A, Zanotti M, Torres J, et al. Histologic grade and perineural invasion are more important than tumor thickness as predictor of nodal metastasis in penile squamous cell carcinoma invading 5 to $10 \mathrm{~mm}$. Am J Surg Pathol. 2008;32(7):974-979.

25. Cubilla AL Meijer CJ, Young RH. Morphological features of epithelial abnormalities and precancerous lesions of the penis. Scand J Urol Nephrol 2000;(205):215-219.

26. Velazquez EF, Piris A, Cubilla. A high grade squamous intraepitelial lesion of the penis:morphologic subtypes corralate with homologous variants of invasive squamous cell carcinoma. a report 126 cases. Mod Path. 2001;14: 126.

27. Lont AP, Kroon BK, Gallee MP, van Tinteren H, Moonen LM, Horenblas S. Pelvic lymph node dissection for penile carcinoma: extent of inguinal lymph node involvement as an indicator for pelvic lymph node involvement and survival. J Urol. 2007;177(3):947-952.

28. Hegarty PK, Kayes O, Freeman A, Christopher N, Ralph DJ, Minhas S. A prospective study of 100 cases of penile cancer managed according to European Association of Urology guidelines. BJU Int. 2006;98(3):526-531.

29. Novara G, Artibani W, Cunico SC, De Giorgi G, Gardiman M, Martignoni G, et al. How accurately do Solsona and European Association of Urology risk groups predict for risk of lymph node metastases in patients with squamous cell carcinoma of the penis? Urology. 2008;71(2):328-333.

30. Bevan-Thomas R, Slaton JW, Pettaway CA. Contemporary morbidity from lymphadenectomy for penile squamous cell carcinoma: The M. D. Anderson Cancer Center experience. J Urol. 2002;167(4):1638-1642.

31. Heyns CF, Theron PD. Evaluation of dynamic sentinel lymph node biopsy in patients with squamous cell carcinoma of the penis and palpable inguinal nodes. BJU Int. 2008;102(3):305-309.

32. Hadway P, Smith Y, Corbishley C, Heenan S, Watkin NA. Evaluation of dynamic lymphoscintigraphy and sentinel lymph-node biopsy for detecting occult metastases in patients with penile squamous cell carcinoma. BJU Int. 2007; 100(3):561-565.

33. Kroon BK, Horenblas S, Lont AP, Tanis PJ, Gallee MP, Nieweg OE. Patients with penile carcinoma benefit from immediate resection of clinically occult lymph node metastases. J Urol. 2005:173(3):816-819.

34. Johnson DE, Lo RK. Management of regional lymph nodes in penile carcinoma. Five-year results following therapeutic groin dissections. Urology 1984;24(4):308-311.

Correspondencia autor: Dr. Carlos Di Capua Sacoto

Servicio de Urología. Hospital Universitario La Fe

Avda. Campanar, 21- 46009 Valencia

Tel.: 963862700

E-mail autor: carlosdicapua@hotmail.com

Información artículo: Original - Cáncer de pene

Trabajo recibido: octubre 2008

Trabajo aceptado: noviembre 2008 